\title{
Miranda
}

Revue pluridisciplinaire du monde anglophone /

Multidisciplinary peer-reviewed journal on the English-

speaking world

$8 \mid 2013$

In Umbra Voluptatis : Shades, Shadows, and their

Felicities / Film Adaptations, New Interactions

\section{Le tâtonnement dans Reader's Block (1996) de David Markson}

Mathieu Duplay

\section{OpenEdition}

Journals

Édition électronique

URL : http://journals.openedition.org/miranda/3638

DOI : $10.4000 /$ miranda.3638

ISSN : 2108-6559

Éditeur

Université Toulouse - Jean Jaurès

Référence électronique

Mathieu Duplay, « Le tâtonnement dans Reader's Block (1996) de David Markson », Miranda [En ligne], 8 | 2013, mis en ligne le 28 juin 2013, consulté le 16 février 2021. URL : http://journals.openedition.org/ miranda/3638; DOI : https://doi.org/10.4000/miranda.3638

Ce document a été généré automatiquement le 16 février 2021.

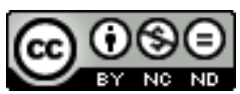

Miranda is licensed under a Creative Commons Attribution-NonCommercial-NoDerivatives 4.0 International License. 


\title{
Le tâtonnement dans Reader's Block (1996) de David Markson
}

\author{
Mathieu Duplay
}

1 A plus d'un titre, Reader's Block (1996) de David Markson est un roman de l'ombre. Empreint d'un lyrisme intense, voire autobiographie déguisée d'un écrivain de plus en plus préoccupé, au terme de sa vie, par l'expérience de la finitude (Palleau-Papin, 18), ce texte à la tonalité parfois presque confessionnelle tente néanmoins le pari paradoxal de rendre le sujet, sa voix, son discours aussi discrets que possible, d'inventer une écriture de la subjectivité qui pourtant renvoie la subjectivité dans l'ombre, dans les coulisses d'une narration où prédomine la rhétorique de l'impersonnel. Insaisissable, le sujet n'est ici, du reste, que l'ombre de lui-même; soumis à un processus de déperdition et d'usure qui le prive peu à peu de toute consistance, il semble menacé de disparaître tout à fait à mesure que la narration chemine vers la limite au-delà de laquelle n'en subsistera plus aucune trace identifiable. Fuyant, insubstantiel, d'ores et déjà réduit au statut de vestige soustrait in extremis - et pour combien de temps encore ? - à l'anéantissement, le sujet subsiste tant bien que mal à la lisière du néant, infime et obsédant résidu qui sépare le «quelque chose » du «rien »; ombre déjà presque dépourvue d'existence, il ne relève plus de l'ontologie, mais d'une « hantologie», pour reprendre le mot de Derrida ${ }^{1}$. Pour preuve, l'obsession de la mort volontaire qui caractérise ce roman où, sans autre commentaire, sont longuement énumérés sur deux pages (189-190) les grands suicidés de la littérature, de Phèdre à Quentin Compson en passant par James O. Incandenza (personnage de Infinite Jest [1995], le texte majeur de David Foster Wallace) : il y a là sans doute une manière de marquer que l'écriture vise à l'extinction délibérée du moi, et qu'en même temps elle se donne pour tâche d'en conserver une trace lisible a minima, à l'instar d'une inscription funéraire. Objet d'une narration possible, le suicide est aussi - d'abord sans doute - la clef d'une poétique, une dimension de l'écriture, indépendamment des événements que le récit évoque ; en témoigne l'importance accordée dans Reader's Block à la figure d'Emily Dickinson, qui n'eut pas besoin de mourir pour hanter sa demeure à la manière d'un spectre.

At Amherst, ca. 1880:

Emily, you wretch! None of this nonsense! I've traveled all the way from Springfield 
to see you. Come down at once. (123)

Roman de l'ombre, Reader's Block l'est encore pour une autre raison, puisque les mots eux-mêmes n'y semblent subsister qu'à titre provisoire, à la lisière de l'invisible, expression qu'il convient en l'occurrence de prendre au pied de la lettre. Comme son titre l'indique, ce livre élabore une phénoménologie de la non-lecture, un inventaire paradoxal des formes sensibles que prend l'entreprise herméneutique lorsqu'elle se sait condamnée à l'échec; il a pour principal, sinon pour seul enjeu narratif ce qui fait de tout déchiffrement un combat avec l'illisible, à commencer par sa propre réception. Parmi les forces hostiles à la lecture, il place au premier rang la perte de lumière, autrement dit de ce qui rend matériellement possible la vision.

At modest estimate, at least 40 percent of the entire population of Europe was annihilated during the four years of the Black Death.

[...] I would give you some violets, but they withered all, when my father died.

Vanishing Point.

Toward what final grievous contemplation amid the disarray?

The sun will run out of hydrogen and commence to die in approximately one billion, one hundred million years. (192)

2 Appelé à s'éteindre, le soleil n'éclaire plus qu'à peine un univers orphelin où la couleur elle-même s'étiole, comme les violettes d'Ophélie, et où l'humanité se prépare à plonger dans les ténèbres d'une nouvelle Mort Noire. Certes, tout n'est pas perdu, du moins pour l'heure : il reste plus d'un milliard d'années avant l'extinction cosmique ; soixante pour cent des Européens ont survécu à la terrible épidémie, et si Ophélie pleure la mort de son père, elle demeure pour sa part en sursis, même si le lecteur n'ignore pas que son suicide est proche. C'est bien l'ombre que le texte évoque, et non pas la nuit - un entre-deux crépusculaire déjà vécu comme un après-coup, puisque l'œil est contraint de s'accommoder à une lumière amenuisée, et pourtant marqué par une interminable attente, par l'anticipation de l'inexorable, mais aussi de ce qui, à l'échelle de l'histoire humaine, ne se produira sans doute jamais, puisqu'il est vraisemblable que notre espèce aura disparu bien avant que le soleil n'épuise ses réserves d'hydrogène. Aussi la lettre, encore visible à la limite sur une page dévorée par l'obscurité croissante, subsiste-t-elle ici à l'état de spectre, à l'instar du sujet censé lui donner sens.

3 La ressemblance est trop frappante pour être fortuite. De fait, ce double mouvement de retrait, ces deux modalités simultanées de l'exposition à l'obscur appellent une explication croisée qui en fait apparaitre la complémentarité.

4 Si l'ombre gagne le texte, suppose-t-on d'abord, c'est peut-être parce que tout incite à se demander s'il existe encore des lecteurs capables de lui donner un sens, ce qui incite à tenir l'évocation cataclysmique du moment où le soleil s'éteindra pour la figure ou la contrepartie objective de la crise qui frappe la subjectivité. La lumière existe-t-elle encore dès lors que « je » ne suis plus là pour la voir? « In the end one experiences only one's self. / Said Nietzsche » (193), affirmation d'où l'on peut déduire que la disparition progressive des caractères d'écriture a pour cause ultime l'incapacité du sujet à se porter garant de leur déchiffrement. Chez Markson, personne (ou presque) n'écrit, au sens fort du terme ; les écrivains, ou ceux que l'on appelle ainsi, ne sont pour la plupart que des lecteurs comme les autres, et rien ne les autorise à revendiquer une position privilégiée vis-à-vis des textes. "First and foremost, I think of myself as a reader ", lance Borges dans la citation donnée en exergue au roman; le narrateur lui fait écho dans une formule à la fois lapidaire et empreinte de modestie : «Joyces write. Readers read » (65), clef de cet étrange récit qui a pour protagoniste un romancier nommé Reader. Écrire, ce n'est le plus souvent qu'aller au bout de sa lecture, explorer sans 
restrictions les potentialités dont elle est porteuse ; or si les écrivains célèbres auxquels se réfère le narrateur de Reader's Block donnent par conséquent une idée claire de ce que lire peut signifier, c'est avant tout sous la forme d'un exemple à ne pas suivre. Suicidaires, débauchés, égocentriques, sujets à divers troubles mentaux, ils n'apparaissent en rien comme des modèles, d'autant qu'ils ne brillent guère par leurs qualités humaines; le roman se présente notamment comme une longue liste d'auteurs antisémites, d'Emmanuel Kant à Ezra Pound en passant par Edith Wharton et Katherine Anne Porter, tous coupables d'avoir cédé à une perversion du sens éthique dont ce texte où passe plus d'une fois le souvenir de la Shoah souligne implicitement l'extrême gravité. Surtout, ces écrivains à la moralité douteuse dépendent de l'œil et de ses fragiles capacités perceptives ; c'est en tout cas ce que suggère l'insistance avec laquelle le texte se réfère à l'histoire de la peinture, comme pour indiquer que la littérature aussi relève à sa manière de ce que l'on appelle en anglais visual arts :

Rosalba Carriera was blind in the last years of her life.

Mary Cassatt was blind in the last years of her life. (105)

Avant d'être une crise herméneutique, le blocage de la lecture, reader's block, traduit la perte progressive du contact avec l'écrit dans sa dimension matérielle, l'incapacité croissante à reconnaître que des inscriptions existent et donc, a fortiori, à se faire une idée de ce qu'elles peuvent signifier. Activité humaine, la lecture est prisonnière, dans Reader's Block, des limites de l'humaine condition, ce qui la rend d'abord tributaire du corps, de sa finitude et de ses faiblesses.

D'autre part, si l'œil peine de plus en plus à percevoir l'écrit, c'est que celui-ci se caractérise par son infinie fragilité : se définir comme «lecteur", voire recevoir pour mission, à la faveur d'une antonomase, d'incarner le «Lecteur» («Reader») par excellence, c'est faire dépendre sa survie de quelque chose qui ne se rencontre que de manière exceptionnelle et sur quoi pèse à tout moment la menace de l'anéantissement. "Throughout the Middle Ages, often no more than a single manuscript of certain classics existed. One leaking monastery roof and the Satyricon could have been lost forever, for instance.» (14) Spectrale, la lettre surgit de l'ombre parce qu'elle se donne à voir sur fond d'une perte irréparable et à l'instant qui précède sa disparition imminente, parce qu'il est de la nature du texte de se présenter comme une trace au second degré, comme l'empreinte vestigielle et précaire d'une inscription en grande partie disparue. Cela ne rend pas l'écriture indéchiffrable, puisque sa fonction de renvoi est préservée, et avec elle son appartenance à l'ordre de la signification ; mais si elle signifie quelque chose, c'est avant tout l'indéfini, l'espace laissé béant par l'absence définitive de ce qui, dès lors, relève de l'inconnaissable. Ainsi, dès lors que « je » choisis d'associer mon destin à celui de l'écrit, ou plus généralement au devenir d'une marque (forcément fragile) laissée sur un support (forcément friable), «je » m'interdis de définir mon identité autrement que comme une modalité singulière du rapport à l'indéterminé. Readers's Block évoque notamment l'exemple de l'enregistrement discographique, empreinte labile de voix depuis longtemps éteintes dont il ne ressort in fine aucune connaissance certaine, excepté celle de la marge d'incertitude qui affecte toute tentative de reconstituer mentalement le passé dont elle témoigne.

7 A recording of Bella figlia dell'amore, with Beniamino Gigli, Amelita Galli-Curci, Louise Homer, and Giuseppe de Luca. Dated December 1927.

Ergo, by one chance in thirty-one, possibly recorded on the day Protagonist was born (167). 

l'on perçoit aussitôt la résonance cartésienne d'une formulation qui rappelle le célèbre énoncé du cogito. Cela dit, le raisonnement de Descartes est en l'occurrence soumis à un renversement complet, puisqu'il ne s'agit pas d'isoler, au sein de l'incertain, une certitude première inséparable de la conscience immédiate que le sujet a de lui-même, mais au contraire de construire sur des bases statistiques un rapport au simple possible (" possibly »), à l'éventualité d'un fait à jamais insaisissable, source de questions sans réponse sur les plus lointaines origines de l'existence subjective, ou de ce qu'il en reste.

l'expérience, de l'experimentum linguce non moins que de l'appréhension réflexive dont dépend la connaissance de soi, comme le laisse entendre la citation (supposée) de Nietzsche, «In the end one experiences only one's self» (193). Non que l'expérience, ainsi définie, soit désormais compromise : au contraire, tout indique que quelque chose reste à la fois lisible et articulable à un vécu subjectif, si fuyant soit-il. La difficulté vient de ce que, quoique possible, elle est désormais inutile, que le rapport au monde, au langage et au soi ne livre plus les clefs de l'existence et qu'il n'en résulte aucun acquis digne d'être préservé, autrement dit plus aucune "expérience», entendue cette fois comme un synonyme d'« apprentissage ». De quelque côté qu'il se tourne, le «je » n'a accès à rien qui ne soit précaire, incertain et fluctuant, à rien qui prémunisse contre l'angoisse de la mort, à rien donc qui soit thésaurisable. Marquée au sceau du paradoxe, l'écriture de Markson traduit l'enfermement solipsiste dans un moi évanescent, instable, dont la fluidité ne dément en rien la finitude puisqu'au contraire elle en est l'expression la plus directe: "je » suis seul avec moi-même, c'est-à-dire avec les multiples fantômes d'inspiration littéraire dont ce moi est constitué, et dont aucun ne m'est du moindre secours. "Protagonists's bleakest conjecturings that he would finish out his life alone » (188), Did it ever, once, enter even s'interroge le texte à propos d'un personnage conçu comme la projection imaginaire de Reader, qui pourtant ne lui tient pas compagnie ni ne dialogue avec lui parce qu'il est lui-même un amas de déchets sans consistance. «Reader's mental wastebasket, a thought for » (84) - or cette " poubelle mentale » pourrait bien, en dernière analyse, s'avérer identique au moi dans sa totalité, ainsi que le suggèrent les dernières lignes du roman (193).

Cette persistance d'un tragique sans résolution (Palleau-Papin, 24) situe Reader's Block dans le paysage littéraire du modernisme tardif (et non d'une postmodernité réconciliée avec l'expérience de l'indécision et du non-totalisable, comme on peut être tenté de l'affirmer un peu vite sous prétexte que l'écriture de Markson, délibérément fragmentaire, illustre une esthétique de l'hétéroclite: «Nonlinear. Discontinuous. An assemblage » [193]). Encore faut-il préciser, en écho à Walter Benjamin, que le modernisme dont il s'agit se présente comme une réaction à l'essentiel dénuement d'une humanité désormais pauvre en expérience et donc tentée par une forme de barbarie, comme séduite par l'idée de la table rase. "La pauvreté en expérience (Erfahrungsarmut) : cela ne signifie pas que les hommes aspirent à une expérience nouvelle », écrivait Benjamin en 1933.

11 Non, ils aspirent à se libérer de toute expérience quelle qu'elle soit, ils aspirent à un environnement dans lequel ils puissent faire valoir leur pauvreté, extérieure et finalement aussi intérieure, à l'affirmer si clairement et si nettement qu'il en sorte quelque chose de valable. (Benjamin 1933, 371) 
Dans Reader's Block plane, on l'a dit, la menace de la cécité, la crainte d'une atteinte de la vision qui, de l'extérieur, mettrait un terme à la lecture et donc à l'écriture. S'y exprime tout aussi clairement la prise de conscience que l'activité de l'œil même parfaitement sain consiste à renoncer délibérément à tout voir, que contempler, lire, écrire sont des activités qui ne se distinguent pas de la recherche d'un aveuglement volontaire, analogue à une forme d'anesthésie. Parmi les nombreux récits discontinus et allusifs que le roman entrecroise, figure l'histoire de la perspective depuis son invention, évoquée par une célèbre citation de Paolo Uccello ( $" O$ che dolce cosa è questa prospettiva!» [182]), via les jeux formels qu'elle permet, par exemple dans les Époux Arnolfini de Jan Van Eyck (28), jusqu'à son démantèlement par les peintres cubistes, dont témoignent les Demoiselles d'Avignon (73). Or si la perspective constitue l'un des principaux dispositifs grâce auxquels l'Occident s'est attaché, depuis la Renaissance, à rendre compte du visible, elle n'en repose pas moins sur la prise de conscience que construire une image, c'est avant tout choisir où faire passer la frontière entre ce qui sera montré et ce qui sera soustrait au regard, tracer arbitrairement la ligne d'horizon au-delà de laquelle on ne distinguera plus rien, décider de l'endroit où se trouvera le point de fuite, celui où les lignes qui définissent l'espace géométrique de la représentation rencontrent l'infini au contact duquel elles s'abîment dans l'indiscernable. L'expression "vanishing point " figure dans les dernières lignes de Reader's Block (192); elle y acquiert un relief particulier, non seulement parce qu'elle apparaît ainsi comme l'une des clefs de tout ce qui précède, mais aussi parce qu'elle semble annoncer la suite de l'œuvre de Markson, qui choisit d'intituler ainsi son avantdernier roman (paru en 2004) : pour lui, peindre, écrire, lire, c'est en quelque sorte contempler le point de fuite, tourner les yeux vers l'ombre ou l'envers du visible, rappeler que la représentation se fonde avant tout sur le choix de ne pas montrer. Le parallèle possible avec la musique, souvent évoquée dans Reader's Block, confirme que c'est bien l'expérience dans sa totalité qui est en cause, et non la seule vision. «Once, not knowing which of several houses was Bizet's, Saint-Saëns simply stood in the road singing an aria from Les Pêcheurs de Perles ", rapporte le narrateur (18); un peu plus loin, il précise qu'il s'agissait de la cavatine de Nadir, "Je crois entendre encore » (19). On sait que le protagoniste, troublé par la voix d'une inconnue voilée, y évoque le souvenir de sa bien-aimée lointaine :

Je crois entendre encore,

Caché sous les palmiers,

Sa voix tendre et sonore

Comme un chant de ramier !

13 Je crois entendre encore, dit Nadir ; autant dire que, pour l'heure, je n'entends plus : et si je chante à mon tour, c'est pour avouer que rien de ce qui frappe mon oreille ne m'importe aujourd'hui, sauf lorsque j'y discerne, à tort ou à raison, les échos d'une autre voix désormais silencieuse. (De même, lorsqu'il fredonne la célèbre mélodie, Saint-Saëns signale que la seule chose qu'il souhaite entendre, c'est la réponse de son ami Bizet.) Ici, la musique ne se réduit pas à ce qui est audible ; elle est, paradoxalement rendu perceptible à l'oreille, le mouvement par lequel l'attention se tourne vers l'inaudible, vers ce qui, pour l'instant, ne peut être perçu en raison de la distance provisoire que les circonstances ont mises entre l'auditeur et l'objet de son désir. De fait, si cette distance venait à s'annuler, le chant, privé de raison d'être, cesserait aussitôt : vouloir qu'il y ait musique, c'est donc renoncer d'abord à tout entendre, opter pour une forme de surdité, opérer un tri au sein de l'univers sonore entre ce qui sera 
offert à l'écoute et ce que l'on a décidé de lui soustraire temporairement, tout comme l'on s'interdit, sous peine de cacophonie, d'exécuter simultanément un opéra de Bizet et une œuvre de Saint-Saëns.

C'est à cela que Markson doit d'être un auteur moderniste - à sa conscience aiguë des limites de l'expérience, et aussi au pressentiment que ce silence, cette ombre portée sans lesquels il n'y a ni vision, ni audition, leur sont peut-être préférables en dernière analyse. Si la question de la fragmentation se pose à chaque instant dans Reader's Block, c'est d'abord, semble-t-il, à une esthétique de la soustraction que ce roman ressortit : "A novel of intellectual reference and allusion, so to speak minus much of the novel » (61). Déjà employée par Benjamin, la métaphore de la pauvreté réapparaît tout au long du roman, associée moins au manque d'argent qu'à l'évocation d'un dénuement existentiel, d'un amenuisement du rapport au monde et à soi que l'on pourrait assimiler à une forme d'ascèse, si la capacité d'y consentir ne faisait elle aussi partie de ce dont le personnage se trouve dépouillé.

His life evidently static. Alone, seemingly without occupation or achievement, his

means meager.

Emptiness. (11)

En procédant ainsi, le texte ne cherche pas à faire en sorte que le rien se transmue en quelque chose, à la faveur d'un retournement dialectique analogue à une nouvelle création ex nihilo - le propos récurrent sur le caractère dérivatif de toute écriture, abstraction faite des quelques cas exceptionnels au nombre desquels Reader ne figure pas, paraît incompatible avec une interprétation aussi présomptueuse. Plus modestement, il s'agit d'échanger la valeur du rien et celle de l'être, de revaloriser le rien au détriment de son contraire, d'«affirmer [la pauvreté] si clairement et si nettement qu'il en sorte quelque chose de valable", comme le propose Benjamin. "Why is there anything at all rather than nothing? » interroge le narrateur de Reader's Block, en écho à la célèbre question de Leibniz (13)-reprise que l'on est enclin à qualifier de parodique, puisque le roman dans son ensemble laisse entendre qu'il est absurde de se préoccuper sans cesse de ce fameux «quelque chose» alors qu'à tout prendre le rien vaut peut-être mieux. "The world is my idea », déclarait Schopenhauer, lui aussi cité par le narrateur (11). Certes ; mais alors qu'arriverait-il si jamais je cessais, comme on dit, de me faire des idées? Si je cessais de penser le monde, ce que l'on appelle un monde continuerait-il d'exister? Et si d'aventure il fallait répondre par la négative, cela serait-il bien grave ? Lors de l'entretien qu'il m'a accordé en septembre 2008, Markson m'a expliqué de quelle manière il a composé ce roman et ceux qui lui ont succédé, à partir de fragments prélevés dans des livres qu'il lisait en diagonale avant de les revendre à des libraires d'occasion. Confirmé par d'autres témoignages (PalleauPapin, 204), cet aveu incite à considérer Reader's Block comme le fruit d'un renoncement volontaire aux textes et à leur lecture suivie, et les citations fragmentaires dont le texte est émaillé non pas comme des reprises intertextuelles à l'origine d'effets de sens, mais comme les vestiges d'un original délibérément perdu: procéder comme le faisait Markson, c'est solder sa bibliothèque, dans tous les sens du terme, autrement dit en diminuer la valeur commerciale et régler définitivement son cas, pour solde de tout compte. Si provocante que soit cette démarche, elle n'égale pourtant pas en radicalité celle de Kate, la narratrice de Wittgenstein's Mistress (1988), qui arrache les pages de son édition d'Euripide et les jette au feu l'une après l'autre au fur et à mesure de sa lecture (Markson 1988, 39). 

d'interpréter ainsi le propos de Benjamin sur la «barbarie» moderne. Certes, Kate détruit les livres des autres, mais cela ne l'empêche pas, dans le même temps, de composer le sien jour après jour : Wittgenstein's Mistress est un Künstlerroman, un roman sur la création littéraire et ses aléas, tout comme Reader's Block qui a pour protagoniste un écrivain.

17 C'est la même attitude que l'on retrouve quand le poète Bertolt Brecht note que le communisme consiste dans la juste répartition, non pas de la richesse, mais de la pauvreté [...]. Un artiste aussi complexe que le peintre Paul Klee, un artiste aussi programmatique qu'Adolf Loos - tous deux repoussent l'image traditionnelle, noble, solennelle, d'un homme paré de toutes les offrandes sacrificatoires du passé, pour se tourner vers leur contemporain qui, dépouillé de ces oripeaux, crie comme un nouveau-né dans les langes sales de cette époque. (Benjamin 1933, 367-368)

Choisir la pauvreté, au sens où Benjamin entend ce terme, c'est refuser de s'abandonner à la nostalgie d'un univers en cours de démantèlement, c'est privilégier ce qui, dans les termes qui avaient cours quand il était encore florissant, s'appelle le rien. Cela dit, le rien n'est pas identique au néant, au contraire, puisqu'il rend possible une forme de reconstruction sociale sur la base d'un partage égalitaire, comme le suggère l'allusion au communisme. Un monde disparaît, celui de la richesse, autrement dit de la culture et de ses monuments, ces «offrandes sacrificatoires du passé »; encombré de tout un bric-à-brac inutile qui rappelle l'esthétique rococo (368) ou les intérieurs surchargés des années 1880 (369-370), il donnait l'exemple de la barbarie au sens le plus négatif du terme, et c'est à lui que fait référence la remarque cinglante que lance Benjamin en 1940 dans « Sur le concept d'histoire » : « il n'est pas un témoignage de culture qui ne soit en même temps un témoignage de barbarie " (Benjamin 1940, 433). A sa place, se fait jour une conscience du contemporain, c'est-à-dire le pressentiment d'un temps partagé, l'attention portée à la condition commune des êtres vivants, unis dans un même dépouillement que figure le vagissement du nouveau-né. D'avance, Benjamin prend ici le contre-pied du propos développé en 1929-1930 par Heidegger, pour qui l'animal est " pauvre en monde » (« weltarm») alors que l'humain est créateur de monde («weltbildend») (267). Selon Benjamin, le barbare renonce à la Bildung («weltbildend»), terme qui désigne en allemand la culture dans son rapport à l'éducation, à la formation de soi ; mais c'est pour découvrir dans la pauvreté animale du bébé emmailloté dans ses « langes sales ", dans cet en -deçà de tout apprentissage, la clef du seul monde aujourd'hui possible. Ou, pour le redire en des termes plus proches de ceux qu'emploie le narrateur de Reader's Block: le salut de l'humain passe par le renoncement complet à l'anthropocentrisme que préfigurent par exemple les allusions récurrentes à la poésie imagiste de William Carlos Williams, auteur pourtant connu pour son attachement aux « choses » (« No ideas but in things », cité p. 67). «Why is the red wheelbarrow beside the white chickens rather than vice versa? " s'interroge le narrateur (92)-question faussement naïve dont il ressort que le regard de Williams se fixe d'abord sur la brouette rouge, objet manufacturé et, à ce titre, synecdoque de la Bildung: si tant est que l'on se fie à ce qu'en dit le texte de Reader's Block, c'est grâce à cette « offrande sacrificatoire » que ce poème en apparence tourné vers le spectacle de l'impersonnel renie son projet explicite et monnaie l'accès subreptice à un nouvel apprentissage de la subjectivité. 
Dépouiller l'homme de ses privilèges "traditionnels", des oripeaux de sa trompeuse «noblesse», et du même coup le faire renoncer à la culture du «je », à l'existence subjective conçue non comme un donné naturel mais comme l'effet d'un savoir-faire acquis, telle est chez Markson la tâche de l'écriture. Trop souvent, les textes s'encombrent de toutes sortes d'accessoires inutiles («baggage », disait déjà Kate ${ }^{2}$ ), ce qui les condamne dans une situation où tout ce qui ne répond pas à une nécessité vitale apparaît ipso facto nuisible: on ne doit pas hésiter à s'en défaire s'ils se montrent incapables d'accomplir leur mission, sous peine de faire le jeu de la barbarie dans ce qu'elle a de plus destructeur. Cela dit, les mêmes raisons imposent de continuer à écrire, car seuls de nouveaux livres pourront donner accès au monde nouveau, si tant est que l'idée même d'un "monde » unifié et ordonné a priori conserve sa pertinence dans ce nouveau contexte. Continuer à écrire, oui, mais pas dans la vive lumière d'après l'orage grâce à laquelle, chez Williams, se détachent la silhouette rouge vif de la brouette et le plumage éclatant des poulets ; l'ombre sied mieux à une telle entreprise. Reader's Block se réfère de manière quasi simultanée à Milton, le poète aveugle de Paradise Lost, et à Rodrigo de Triana, le premier à avoir aperçu l'Amérique depuis la Pinta, un petit matin d'octobre 1492 :

October 12, 1492. At 2 A.M., the lookout on the Pinta.

Named Rodrigo de Triana.

No light; but rather darkness visible. (99)

20 A rebours des clichés millénaristes qui dépeignent le continent ainsi découvert comme un nouvel Eden auquel conviendrait mieux une lumière céleste, le texte lui associe une description du paysage infernal tel qu'il apparaît à Satan peu après sa chute (I.63). Cela dit, l'instant du premier regard lancé à travers l'obscurité n'en marque pas moins, à une échelle plus modeste, un bouleversement salvateur qui donne accès à une autre dimension du réel, via une transformation paradoxale de la perception («darkness visible »). L'enjeu est le suivant : dire non, tel Satan, à un prétendu Paradis où l'on ne reconnaît plus rien qui ressemble de près ou de loin à une solution; dire non, du même coup, à la dramaturgie épique de la Rédemption que le christianisme élabore à partir des textes bibliques et à laquelle, à un certain niveau, le texte miltonien apporte son concours. Pourtant, cela n'interdit pas d'adhérer à une tout autre conception du messianisme, que celle-là ne rencontre nullement. Espoir du salut par l'imperceptible, renoncement aux illusions inhérentes à l'expérience sensible, recherche d'un aveuglement volontaire qui est cécité à ce qui fait obstacle, à ce qui gêne la vision, autrement dit, au sens étymologique du terme, à l'objet sur lequel le regard vient buter, ce messianisme d'un autre genre encourage l'ouverture à l'illimité et prépare un renoncement à l'ego, un « communisme » au sens de Benjamin et de Brecht, inséparable d'une pratique de l'impersonnel.

Dès lors se pose la question du tâtonnement. Comment se comporter - et singulièrement comment lire - dans un monde où l'on n'y voit rien, au propre comme au figuré ? En mobilisant les autres sens à titre de compensation, est-on tenté de répondre dans un premier temps; c'est sans doute pour une part ce qui justifie la place accordée à la musique dans ce roman, expression d'une réceptivité accrue, dans un monde crépusculaire, à ce que l'œil ne voit pas ou voit mal. Pourtant, ces efforts ne suffisent pas : l'ouïe la plus affûtée ne se substitue jamais tout à fait à la vision manquante, et l'on est bien obligé d'y aller à tâtons. Au figuré, tout d'abord, parce qu'une telle démarche privilégie la recherche expérimentale de solutions empiriques et 
encourage un nouvel apprentissage qui, faute de pouvoir s'appuyer sur une expérience préalable, procède de manière pragmatique, sans exclure l'imperfection ni l'erreur.

None of John Milton's daughters was given an education, though two of the three were taught to read to him in his blindness.

In languages of which they did not understand one word. (16)

L'incompréhension, l'ignorance ne font pas obstacle à la lecture ainsi comprise; au contraire, elles en sont la condition de possibilité, car la pauvreté dont elles sont le signe donne accès à la cécité visionnaire sans laquelle il n'y a pas de texte qui vaille. Au figuré, mais aussi au propre, parce que les transformations de la perception appellent un travail sur le corps qui confère une importance nouvelle à la main, organe sensoriel mais aussi source d'une interrogation radicale sur les rapports entre pensée et action. On a vu que dans Reader's Block, le tragique a pour origine les hésitations inhérentes à l'expérience du corps, les incertitudes que comporte un rapport aux mots tout entier fondé sur la relation fragile entre un œil en voie d'affaiblissement et l'écrit dans sa matérialité précaire ; il naît d'une résistance affirmée à la dématérialisation du savoir, du refus de distinguer les textes de leurs supports concrets, mais aussi de la volonté délibérée de rappeler que l'acte cognitif est soumis aux aléas de la chair, figurés notamment par la vieillesse et par la mort. Ici, l'hybris tragique consiste à croire que le savoir - celui, par exemple, de la littérature - appartient à la dimension de l'idéalité, alors qu'il part du corps et n'a de cesse d'y reconduire, voire ne s'en affranchit jamais. Balzac le savait bien, lui pour qui la littérature n'avait pas d'autre fonction que de diagnostiquer les maladies de la chair, et pourquoi pas d'y remédier :

Only Bianchon can save me, said Balzac, near death.

Bianchon being a doctor in Le Père Goriot. (11)

Aussi ne peut-on tirer les leçons de cette situation qu'en recourant à un mode corporel d'appréhension des mots qui ne privilégie pas d'emblée la quête de l'abstraction, à la différence de la vision, que la tradition philosophique associe à la theoria, à la contemplation intellectuelle du vrai. Surprendre et humilier l'esprit, tel est l'enjeu; il s'agit de lui faire comprendre qu'il est constamment tenté de s'aveugler sur sa véritable nature, sur l'origine et les finalités de la pensée. Pour cela, il n'est pas inutile de lui rappeler qu'il a des liens peut-être plus intimes avec la main qu'avec l'œil : «Why is Reader always mildly incredulous at remembering that the decimal system arose from counting on one's fingers? » (16) La pensée n'est pas un regard tourné vers le ciel des Idées ; au contraire, elle cherche à s'emparer du donné concret, elle se donne pour but de manier les choses et s'avère à ce titre inséparable de l'action. Si l'on peut trouver ici un écho du pragmatisme de William James, c'est peut-être avec Wittgenstein que le rapprochement est le plus pertinent, et l'on est tenté d'évoquer The Blue Book: «We may say that thinking is essentially the activity of operating with signs. This activity is performed by the hand, when we think by writing " (6).

Eclairante, cette comparaison l'est notamment parce qu'elle met l'accent sur ce qu'a de libérateur une approche qui dissuade d'associer la pensée à l'intériorité : raisonner ainsi, c'est montrer que l'activité mentale n'est pas l'émanation de ce qui se trame dans les profondeurs de l'être, mais qu'au contraire elle est affaire de surfaces, qu'elle fonctionne comme une machine ("operating with signs ») parce qu'elle procède au rapprochement d'éléments hétérogènes au sein d'un réseau potentiellement illimité car susceptible de s'étendre à la totalité du monde physique. Du point de vue du sujet, qui n'a pas encore perdu le souvenir de ses anciens privilèges, une telle démarche paraît certes austère puisqu'elle ne reconnaît aucun privilège à l'individu considéré 
isolément et va jusqu'à le tenir pour quantité négligeable en dehors des rapports qu'il entretient avec ce qui l'entoure; mais le renoncement à soi que ce raisonnement encourage prend in fine la valeur d'une ascèse salvatrice, d'un exercice spirituel à visée thérapeutique qui affirme la supériorité d'un impersonnel capable d'atteindre aux dimensions du cosmos. Paradoxalement, souligner avec intransigeance la matérialité du corps pensant n'équivaut ni à une forme de nihilisme, ni à une prise de position matérialiste, puisqu'en mettant l'esprit à sa vraie place elle le fait voir dans sa nudité, et l'on aurait tort de voir un geste désespéré dans ce qui traduit au contraire une quête de sainteté :

Simeon Stylites, who spent thirty-six years on top of a sixty-foot pillar in the Syrian desert. For most of that time his body a mass of maggot-infested sores.

The maggots no more than eating what God had intended for them, he said. (55)

A vrai dire, la question ne consiste pas tant à déterminer si une forme de spiritualité est ici en jeu qu'à en définir précisément la nature. Le vocabulaire employé évoque la Providence, l'intention de Dieu, autrement dit une vision téléologique de l'existence humaine qui n'est pas sans rappeler la théologie chrétienne à laquelle le Stylite souscrivait. Cela dit, on observe également que le comportement de l'ascète traduit sa volonté de faire en sorte que ce projet divin se réalise dès aujourd'hui, sans délai : il n'attend pas d'être mort pour donner son corps en pâture à la vermine, ni donc, est-on en droit de supposer, pour laisser son âme rejoindre Dieu; en s'imposant cette pénitence, il invente un nouveau rapport au corps tel que chaque instant, quel qu'il soit, est déjà celui du salut, puisqu'il est celui où s'accomplissent les desseins du Ciel. C'est ainsi que Siméon ouvre la voie à la transformation messianique qu'annonce aussi, on l'a vu, la citation de Paradise Lost, à cette différence près qu'elle se traduit ici par une expérience renouvelée du temps et non par un bouleversement d'ordre sensoriel. Le texte se réfère implicitement à la durée prévisible de l'existence individuelle, et avec elle au déroulement chronologique qui confère une historicité à la vie humaine : « who spent thirty-six years "... Aussitôt convoquée, cette forme classique de temporalité se trouve suspendue par le choix de tournures nominales qui relèguent les verbes conjugués en position de subordination ("Simeon Stylites, who spent...»), ou encore par le recours à la forme progressive qui met l'accent sur le processus au détriment de la succession des événements : " no more than eating ». Au sein d'un récit biographique au déroulement linéaire s'immisce ainsi un autre temps, fait d'une série d'instants à la fois présents et incommensurables parce que l'on n'en connaît ni le commencement, ni la fin.

On reconnaitt ici le temps messianique tel que le décrit Stéphane Mosès :

[...] le temps [n'est] plus pensé comme un axe orienté, où l'après succède inévitablement à l'avant, ou comme un fleuve qui coule de sa source vers son embouchure, mais comme une juxtaposition d'instants chaque fois uniques, non totalisables, et qui, par conséquent, ne se succèdent pas comme les étapes d'un processus irréversible (25-26).

$\mathrm{Si}$, selon Mosès, cette modalité du temps se manifeste par exemple chez Borges, c'est chez Kafka ou encore chez Walter Benjamin, Franz Rosenzweig et Gershom Scholem qu'il s'en rencontre les évocations les plus précises. Que ces auteurs soient juifs, à l'instar de Markson, n'a rien pour lui d'une coïncidence : tous s'attachent à élaborer une pensée de la temporalité qui s'ancre dans l'expérience d'une « rupture irréversible du tissu historique » liée au traumatisme de la Première Guerre mondiale-crise encore aggravée par la Shoah, qui ruine "l'idée du sens de l'histoire» (31), " de toute 
théodicée inhérente à l'histoire » (29). D'une certaine manière, Mosès laisse entendre ainsi que le propos de Benjamin sur la "barbarie ", équivalent de la "pauvreté " ascétique qui caractérise les derniers romans de Markson, dialogue par anticipation avec la célèbre remarque d'Adorno (elle aussi citée dans Reader's Block): «Nach Auschwitz ein Gedicht zu schreiben, ist barbarisch" (61) - "écrire un poème après Auschwitz est barbare » (ma traduction).

En dernière analyse, le corps, le tâtonnement, le temps ne se laissent pas ramener, chez Markson, à des questions d'ordre thématique ou formel ; les véritables enjeux se situent ailleurs, au croisement d'une éthique et, pourquoi pas, d'une mystique de l'écriture, ce dont il est impossible de rendre compte au fil d'une analyse du fait textuel dans sa nudité. Certes, différents aspects de cette problématique complexe sont explicitement évoqués tout au long de Reader's Block; par ailleurs, la temporalité particulière de la narration, les discontinuités qui la caractérisent, son refus de la linéarité, sa manière aussi de construire malgré tout de vastes réseaux de sens illustrent très bien le propos de Mosès. Cela dit, c'est en amont et en aval, dans le travail de la composition et de la réception, que l'essentiel se joue; autrement dit, Reader's Block ne doit pas être lu comme un «texte " au sens post-structuraliste du terme, comme un fait signifiant que l'on doit appréhender indépendamment de son auteur ${ }^{3}$, mais comme le produit d'un processus incarné auquel le corps lisant et écrivant participe en permanence. C'est là, sans doute, l'une des principales originalités de Markson, que l'on aurait bien tort de tenir pour un réactionnaire sous prétexte qu'il accueille avec méfiance certains discours critiques en vogue à l'époque où il écrit ses romans. Chez lui, nul signe d'un retour à une conception romantique de l'écrivain-démiurge, hors de propos dans un contexte où rien n'apparaît plus incertain que ce que subjectivité veut dire. Au contraire, l'enjeu consiste à s'interroger sur la pertinence des délimitations censées séparer le dedans et le dehors du texte, la personne de l'auteur et celle de son lecteur : ce n'est pas en ces termes, suggère-t-il, que l'on peut espérer rendre compte du rapport à l'écrit, qui n'a que faire de pareilles dichotomies. L'écriture en appelle à la main, au corps; oui, mais lesquels? - interrogation troublante dans un contexte où la question " qui ? » paraît entre toutes dénuée de sens. La figure ambiguë de Reader résume à elle seule toutes les hésitations qui en résultent : projection fictive du scripteur, il est aussi un miroir tendu au lecteur qui, à sa manière, participe à un geste d'inscription d'emblée inséparable de la réception, puisqu'il exprime moins la volonté de s'imposer qu'une forme d'assentiment à ce qui échappe au contrôle de l'individu, voire lui dénie toute existence autre que provisoire. De ce point de vue, les évocations du cabinet de travail de l'écrivain qui figurent au début de Reader's Block s'avèrent particulièrement parlantes. «On a shelf beside Reader's desk: a human skull, a reproduction of Giotto's portrait of Dante, two small rough orange stones" (17); "On one of reader's windowsills: framed photographs of his son and daughter, a scuffed baseball, a sunbleached knotted root plucked from the Ebro " (28). Descriptions d'une machine d'écriture, ces passages associent le vivant et le mort, le proche et le lointain, l'écrit et l'image, la matière et la représentation, l'organique et l'inorganique, le regard contemplatif porté sur l'œuvre d'art et le geste du sportif en butte à la résistance du monde, au sein d'un réseau souple et indéfiniment extensible car essentiellement ouvert, ce que signalent les allusions aux enfants de l'écrivain et donc, implicitement, à son imprévisible postérité. Tout cela a la valeur d'un memento mori, comme le suggère la tête de mort, pièce maîtresse de cette vanité moderne, rappel de l'ombre sans laquelle nulle vision n'est possible. Cela dit, par-delà la menace qui pèse sur le sujet, il y a le jeu, 
la balle mille fois lancée et rattrapée au vol, l'impact musculaire du projectile happé à mi-course, les signes d'écriture rugueux comme des cailloux que lecteur et scripteur s'échangent de page en page et qui, dépourvus de valeur intrinsèque, n'ont de prix que parce qu'ils se présentent comme des manifestations tangibles de l'illimité.

\section{BIBLIOGRAPHIE}

Benjamin, Walter. « Expérience et Pauvreté ». 1933. Trad. Pierre Rusch. In Cuvres II. Paris : Gallimard, 2000. 364-372.

---. « Sur le concept d'histoire ». 1940. Trad. Maurice de Gandillac et Pierre Rusch. In CEuvres III. Paris : Gallimard, 2000. 427-443.

Derrida, Jacques. Spectres de Marx. Paris : Galilée, 1993.

Heidegger, Martin. Les Concepts fondamentaux de la métaphysique. 1938. Trad. Daniel Panis. Paris: Gallimard, 1992.

Markson, David. Entretien avec Mathieu Duplay. New York, 4 septembre 2008.

---. Reader's Block. Normal, IL: Dalkey Archive Press, 1996.

---.Wittgenstein's Mistress. Normal, IL: Dalkey Archive Press, 1988.

Mosès, Stéphane. L'Ange de l'histoire. Rosenzweig, Benjamin, Scholem. 1992. Paris: Gallimard, 2006.

Palleau-Papin, Françoise. Ceci n'est pas une tragédie : l'écriture de David Markson. Lyon: ENS Editions, 2007.

Wittgenstein, Ludwig. The Blue Book. 1958. In The Blue and Brown Books. Preliminary Studies for the "Philosophical Investigations." New York: Harper \& Row, 1965.

\section{NOTES}

1. «[Q]u'est-ce qu'un fantôme? qu'est-ce que l'effectivité ou la présence d'un spectre, c'est-à-dire de ce qui semble rester aussi ineffectif, virtuel, inconsistant qu'un simulacre ? Y a-t-il là, entre la chose même et son simulacre, une opposition qui tienne ? [...] Appelons cela une hantologie. » (31). 2. «Still, perhaps there is baggage after all, for all that I believed I had left baggage behind. / of a sort. The baggage that remains in one's head, meaning remnants of whatever one ever knew. / Such as the birthdays of people like Pablo Picasso or Jackson Pollock, for instance, which I am convinced I might still recite if I wished. / Or telephone numbers, from all of those years ago. » (15)

3. «Diegetic, dialogic, hegemonic. Privilege, as a verb. Foreground, as a verb. Valorize. Praxis. Simulacra. Metafiction. Logocentrism. Phallocentrism. Discourse. Signifier/signified. Aporia. / Late capitalism. Gynophobia. / Text. // None of the above ", lance le narrateur de Reader's Block: ce catalogue ironique de clichés post-structuralistes n'incite guère le critique à les reprendre de manière non distanciée. (156) 


\section{RÉSUMÉS}

Roman de l'ombre, Reader's Block de David Markson raconte entre autres choses l'effacement de la subjectivité, réduite à presque rien dans un monde gagné par les ténèbres où l'écriture même, faute de lumière, devient peu à peu indéchiffrable. Ainsi, le blocage de la lecture qu'évoque le titre traduit moins une crise herméneutique qu'il ne résulte d'une incapacité physique, de la perte de contact avec l'écrit dans sa dimension matérielle, comme le rappellent aussi les allusions fréquentes à la cécité. Cette situation donne naissance à un tragique de l'experimentum linguce, clef d'une esthétique d'inspiration moderniste qui a pour maître-mot l'acceptation ascétique d'une pauvreté recherchée d'autant plus volontiers qu'elle est au fond perçue comme libératrice. $\mathrm{Ne}$ plus rien y voir, ne plus rien posséder en propre (à commencer par soi-même), c'est aussi, dans Reader's Block, être en mesure de repartir à tâtons à la découverte des mots, au croisement d'une éthique et d'une mystique de l'écriture qui toutes deux privilégient le contact tactile avec le monde au détriment d'une intériorité dénoncée comme illusoire.

Reader's Block narrates, among other things, the gradual disappearance of subjectivity: reduced to a shadow of itself, it barely subsists in a darkening world where writing is increasingly illegible for lack of light. Thus, the "reader's block» punningly referred to in the title is less an expression of a hermeneutical crisis than the consequence of a physical impossibility, as the many allusions to blindness also suggest. This situation gives rise to a tragic form of experimentum lingux; in turn, this is key to a Modernist aesthetics which promotes asceticism and the deliberate acceptance of an essential poverty understood, in the last analysis, as a form of liberation. In Reader's Block, blindness and dispossession-including the loss of subjectivity and self-are what make it possible to embark on a groping quest for words, guided by an ethical and mystical awareness of language which privileges tactility at the expense of interiority, which is simultaneously denounced as an illusion.

\section{INDEX}

Keywords : reader's block, subjectivity, writing, modernism, poverty, tragedy, language, tactility Mots-clés : reader's block, subjectivité, écriture, modernisme, pauvreté, tragique, langage, tactilité

\section{AUTEURS}

\section{MATHIEU DUPLAY}

Université Lille III

Professeur

mduplay@club-internet.fr 